\title{
Traumatic Hepatic Arterioportal Fistula Caused by Penetrating Injury
}

\author{
Kang Kook Choi, Min A Lee, Dae Sung Ma, Gil Jae Lee, Byung chul Yu, Jung Nam Lee \\ Department of Trauma Surgery, Gachon University, Gil Medical Center, Incheon, Korea
}

Hepatic arterioportal fistula (APF) can be congenital, acquired, or iatrogenic. Trauma-induced APF is a rare pathologic condition. We report the case of a patient with APF accompanied by hepatic ischemia that was successfully treated using angioembolization.

Key Words: Hepatic Artery; Portal Vein; Fistula

(Trauma Image Proced 2017(1):32-34)

\section{CASE}

A 46-year-old male was stabbed in the right chest and transported to the trauma bay. The patient was alert. His initial vital signs were as follows: blood pressure,
100/60mmHg; pulse, 122 beats/min; respiratory rate, 21 breaths/min; and body temperature, $35^{\circ} \mathrm{C}$. Emergency laparotomy was performed, which revealed that hepatic segment 6 was penetrated and had a 6-cm long penetrating wound. Primary suture closure and perihepatic

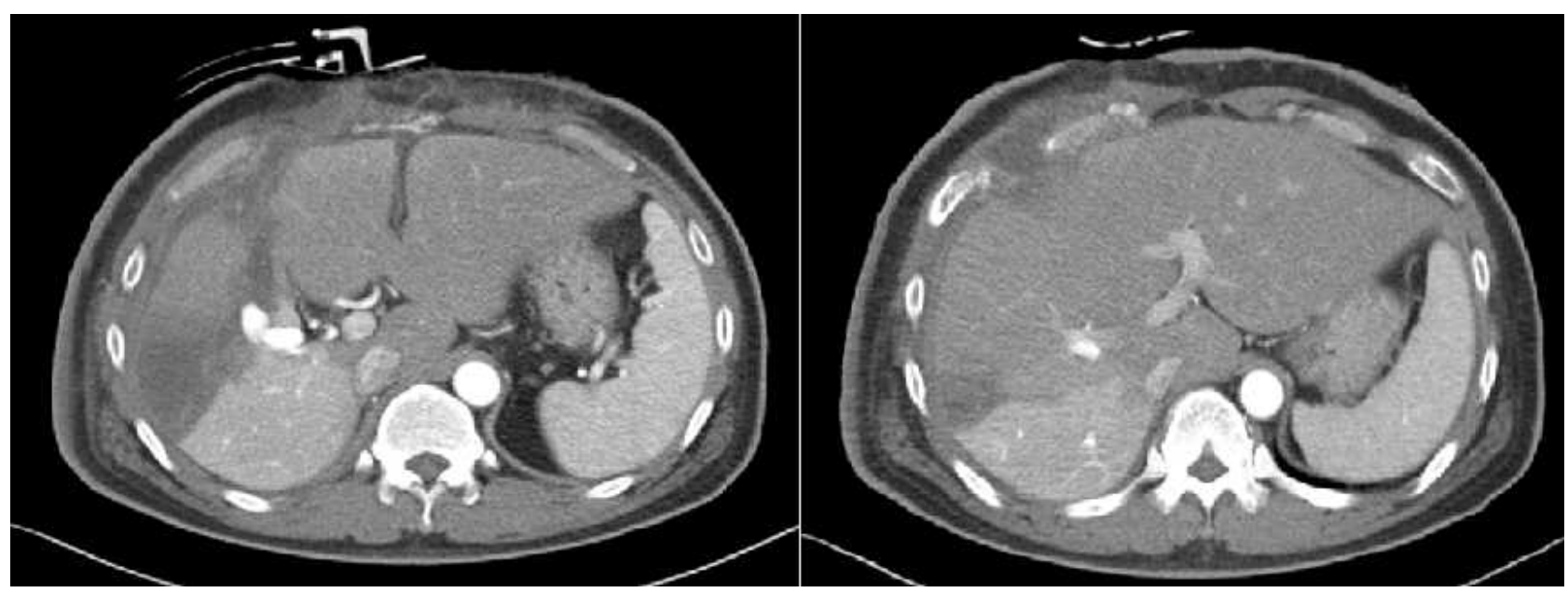

Fig. 1. Abdominal CT scan shows arterioportal venous fistula and pseudoaneurysm on POD\#27. A large ischemic area is also noted.

Received: May 1, 2017 Revised: May 10, 2017 Accepted: May 23, 2017

Correspondence to: Jung Nam Lee, Department of Trauma Surgery, Gachon University, Gil Medical Center, 21, 774 beon-gil, Namdongdaero, Namdong-gu, Incheon 21565, Korea

Tel: 82-51-240-7369, Fax: 82-51-240-7719, E-mail: calvin511@naver.com

Copyright (c) 2017 Korean Association for Research, Procedures and Education on Trauma. All rights reserved.

@This is an open-access article distributed under the terms of the Creative Commons Attribution Non-Commercial License (http://creativecommons.org/ licenses/by-nc/4.0) which permits unrestricted noncommercial use, distribution, and reproduction in any medium, provided the original work is properly cited 

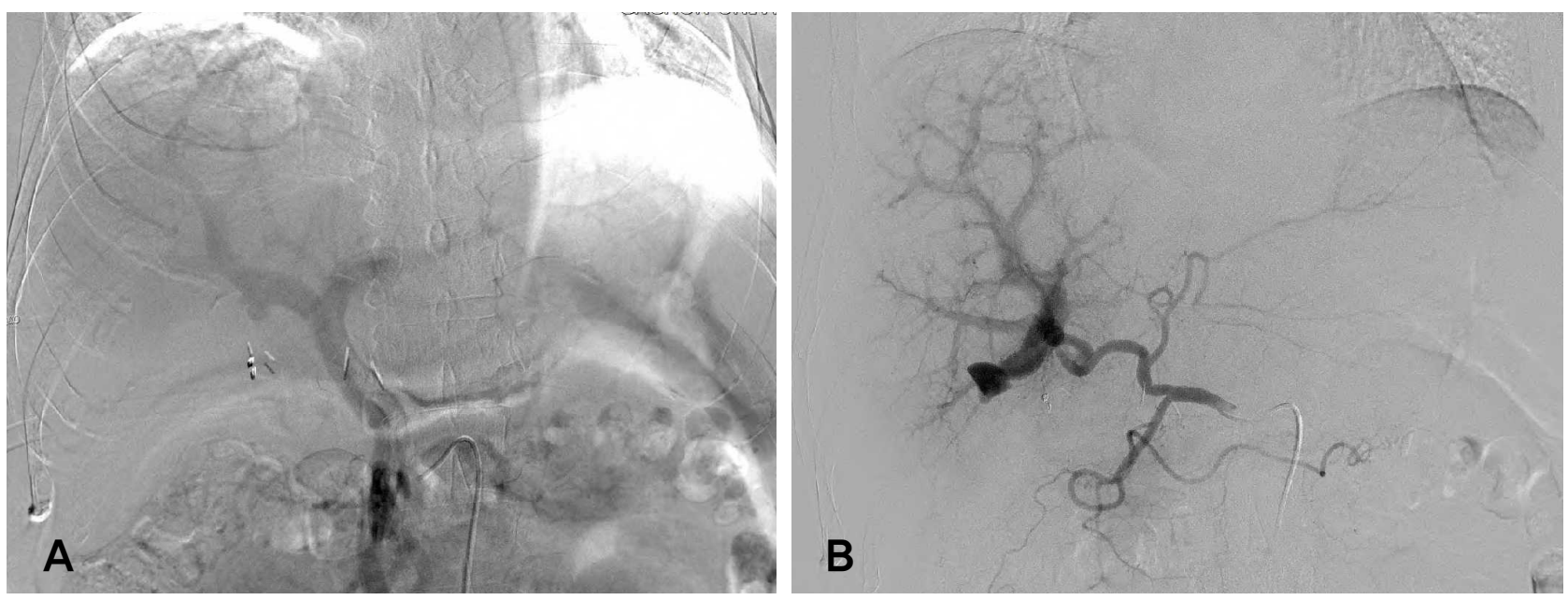

Fig. 2. Hepatic arteriogram shows an arterioportal venous fistula between the right posterior hepatic artery and portal vein (A). The pseudoaneurysm between the hepatic artery and portal vein was successfully occluded by angioembolization using microcoil and glue (B).

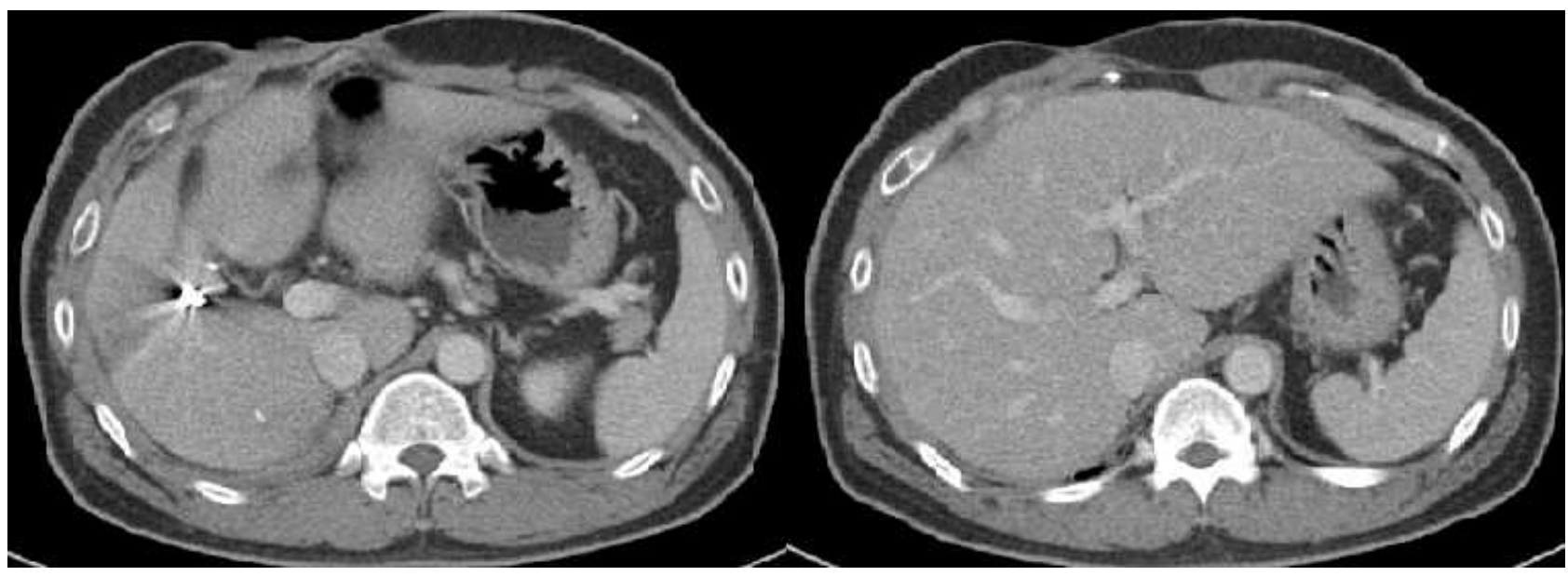

Fig. 3. Abdominal CT performed after 6 months shows disappearance of APF and the ischemic lesion of the liver.

packing of the lacerated liver were performed. One day postoperatively, the patient's vital signs were not optimized, and a second-look operation revealed a newly developed right adrenal gland laceration and perforation of inferior vena cava (IVC). Right adrenalectomy and primary closure of IVC were performed. At 27 days after the initial operation, abdominal CT scan revealed a hepatic APF (Fig. 1.). The APF was successfully occluded by hepatic embolization (Fig. 2.). Abdominal CT repeated 6 months after the operation (Fig. 3.) revealed that the APF and ischemic lesion of the liver had disappeared.

\section{DISCUSSION}

APF can be asymptomatic but can cause complications, such as portal hypertension, which can lead to gastrointestinal bleeding, ascites, heart failure, and intestinal ischemia $(1,2)$. In this patient, the hepatic ischemia might have been a complication of APF. Currently, angioembolization is the treatment of choice for most APFs (1,2). Effective treatment requires expertise in determining the location of the fistula and achieving superselective catheterization and complete occlusion of all feeding arteries. APFs should be 
diagnosed early because complications can be serious and spontaneous resolution is rare. An angiography can confirm the diagnosis $(1,2)$. The angioembolization for the APF is curative in a high percentage of patients with little morbidity.

\section{Conflict of Interest Statement}

No potential conflict of interest relevant to this article was reported.

\section{REFFERENCES}

1. Guzman EA, McCahill LE, Rogers FB. Arterioportal fistulas: introduction of a novel classification with therapeutic implications. Journal of gastrointestinal surgery. 2006;10(4):543-50.

2. Lumsden AB, Allen R, Sreeram S, Atta H, Salam A. Hepatic arterioportal fistula. The American Surgeon. 1993; 59(11):722-6. 